\title{
Optical Phase Refractometer Based on Post-Processed Interferometric Tip Sensors
}

\author{
Marta S. Ferreira, Student Member, OSA, Jörg Bierlich, Sonja Unger, Kay Schuster, \\ José L. Santos, Member, OSA, and Orlando Frazão, Senior Member, OSA
}

\begin{abstract}
An interferometric tip sensor based on the postprocess of a special design double-cladding optical fiber is proposed. Due to the sensing head design, it is sensitive to environmental variations. In order to analyze this effect, the sensing head is subjected to temperature variations both in liquid and gas (at $1 \mathrm{~atm})$. Comparing the two signals, it is possible to discriminate the contribution of the liquid refractive index variation with temperature. Not only the amplitude of the signal varies with the surrounding medium, but also the phase of the interferometric pattern alters. This is due to the presence of a thin diaphragm at the end face of the fiber structure turning the sensing head in a three wave interferometric device. An indirect measurement of the water refractive index is performed, by subjecting the sensing head to temperature variations in air and water. Even though the sensitivities obtained are lower than the ones reported in the literature, it should be highlighted that there is no core exposition of the fiber to the external medium. The sensor is easy to fabricate, robust, and reproducible.
\end{abstract}

Index Terms-Interferometry, post-processing, refractive index measurement.

\section{INTRODUCTION}

$\mathbf{T}$ HE design of Fabry-Perot (FP) type interferometric microcavities in optical fibers has been focus of research not only due to the ease of fabrication and high-reproducibility, but also because they can be highly sensitive to external medium variations. Usually, these sensors are intrinsic, as the cavity is formed inside the optical fiber, and with reduced dimensions (of the order of hundreds of micrometers). These characteristics make them suitable for a wide range of applications like in physics, chemistry, biology, industry, and medicine, among others.

The measurement of refractive index, both of gases and liquids is of great attractiveness when considering these configurations. Usually, for gas measurements, the microcavity is subjected to changes of gas pressure, a parameter that is directly related to the refractive index of the gas. Different configurations

Manuscript received March 5, 2014; revised June 5, 2014; accepted June 30, 2014. Date of publication July 10, 2014; date of current version August 11, 2014. This work was supported in part by the COST Action TD1001 and FCTFundação para a Ciência e Tecnologia under Grant SFRH/BD/76965/2011.

M. S. Ferreira, J. L. Santos, and O. Frazão are with the INESC Porto, 4169007 Porto, Portugal, and also with Faculdade de Ciências da Universidade do Porto, 4169-007 Porto, Portugal (e-mail: msaf@inescporto.pt; josantos@ fc.up.pt; ofrazao@inescporto.pt).

J. Bierlich, S. Unger, and K. Schuster are with the Leibniz Institute of Photonic Technology, 07745 Jena, Germany (e-mail: joerg.bierlich@ipht-jena.de; sonja.unger@ipht-jena.de; kay.schuster@ipht-jena.de).

Color versions of one or more of the figures in this papers are available online at http://ieeexplore.ieee.org.

Digital Object Identifier 10.1109/JLT.2014.2335272 have been reported, using hollow fibers with photonic crystal fibers (PCFs) diaphragms [1], diaphragm-free hollow core fibers [2], or splices with large lateral offsets [3].

Regarding the liquid refractive index measurements, two different approaches have been used: the analysis of visibility or wavelength variations. Sensing heads formed by splicing special fibers between standard single mode fibers (SMF) usually exhibit variation of the visibility with the refractive index modification. These non-standard fibers can be suspended core fibers [4], PCFs [5], multimode fibers combined with hollow core fibers [6], or even hollow core fibers ended with a hollow core silica sphere tip [7]. Another possibility is to use a polymer to form the microcavity at the fiber tip [8]. Even though the sensor presented high resolution, the unprotected tip was very fragile.

Several FP-based sensors have been proposed to measure the wavelength shift with the refractive index variations. Usually, they are obtained by means of post-processing of optical fibers either by writing a long period grating in a PCF section [9], by fabricating microchannels through femtosecond laser micromachining [10]-[12] or by focused ion beam milling [13]. A refractometer based on a microslot engraved along with a fiber Bragg grating has also been reported [14]. In this case, the microslot was obtained through chemically assisted femtosecond laser post-processing.

These kinds of FP microcavities also exhibit very good responses to temperature. Measurements up to $1000^{\circ} \mathrm{C}$ were reported for sensors based in the splices in series of hollow-core fiber, SMF, and PCF [15], as well as post-processed double-clad optical fibers (DCFs) [16] or even micromachining [17].

The measurement of refractive index of liquids through the induced temperature variations has also been a matter of study over the last years. In such cases, the fiber core was exposed to the liquid, resulting in high-sensitive fiber sensors. The core exposition to the liquid was obtained, for instance, by using a large-core air clad PCF, which, due to the large dimensions, had a ring of air holes in contact with the external medium [18]. In this case, a maximum sensitivity of $800 \mathrm{~nm} / \mathrm{RIU}$ was attained. A different configuration was proposed by Wang et al. [19]. They used a simplified hollow-core (SHC) PCF fiber section spliced between two SMFs. Using a femtosecond laser, vertical microchannels were obtained in the SHC PCF, which was then filled with liquids. A sensitivity of $\sim 851.3 \mathrm{~nm} / \mathrm{RIU}$ was achieved with this sensing head.

Another characteristic of these types of sensors is their ability to be multiplexed, allowing different interferometers to be used 


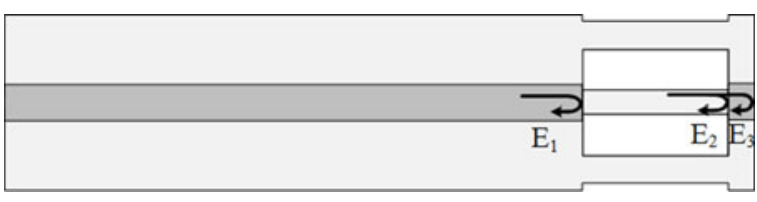

Fig. 1. Fiber microcavity structure evidencing the mirror reflections.

[20] or to measure different parameters [21]. In this case, it is required to use an adequate signal processing for the sensors spatial discrimination. The use of the fast Fourier transform (FFT) was reported, since the intensity of the FFT spectra was linearly related with the refractive index.

In this paper, through the post-processing of a (DCF and the incorporation of a diaphragm, a three-wave interferometric microcavity is proposed. The sensing head is subjected to temperature measurements in gas and liquid. Due to its different responses, it is possible to estimate the contribution of the water refractive index variation with temperature.

\section{Sensing Structure AND OUter Medium}

\section{A. Three-Wave Fiber Microcavity}

The scheme of the fiber microcavity under study is shown in Fig. 1. The sensing head is constituted by a small section of postprocessed DCF and a very thin diaphragm composed by standard SMF (SMF 28). Also shown in the figure are the reflections $\left(E_{1}, E_{2}\right.$, and $\left.E_{3}\right)$ that can occur in the structure, indicating the sensing head has an interferometric behavior associated with the interference of three waves, the first two with fixed amplitudes and phases, while the third one has an essentially fixed phase but an amplitude that depends on the refractive index of the external medium. Here, particularly important is the situation where we are in presence of aqueous environments due to their central role in biochemistry.

The first wave, $E_{1}$, occurs at the interface between the SMFDCF at the input of the cavity (due to the mismatch between the effective refractive index in each side). The second, $E_{2}$, is originated at the end of this cavity by the same effect. Considering that $E_{1}$ has a phase $\varphi_{1}$ when generated in the reflection, the phase difference with $E_{2}$ is

$$
\varphi_{2}=\varphi_{1}+\frac{4 \pi n_{e 2} L_{c}}{\lambda}
$$

where $n_{\mathrm{e} 2}$ is the effective refractive index inside the DCF cavity, $L_{c}$ is the cavity length, and $\lambda$ corresponds to the wavelength.

The third wave $\left(E_{3}\right)$ occurs at the interface between the diaphragm and the external medium. The phase difference of this wave with $E_{2}$ is described by the following equation:

$$
\varphi_{3}=\varphi_{2}+\frac{4 \pi n_{e 1} L_{d}}{\lambda}
$$

where $n_{\mathrm{e} 1}$ is the diaphragm effective refractive index and $L_{d}$ is the diaphragm length. This reflection $(\sim 4 \%$ with air as surrounding), is substantially stronger than the second one, so the interferometric behavior of the cavity is essentially determined by $E_{1}$ and $E_{3}$.
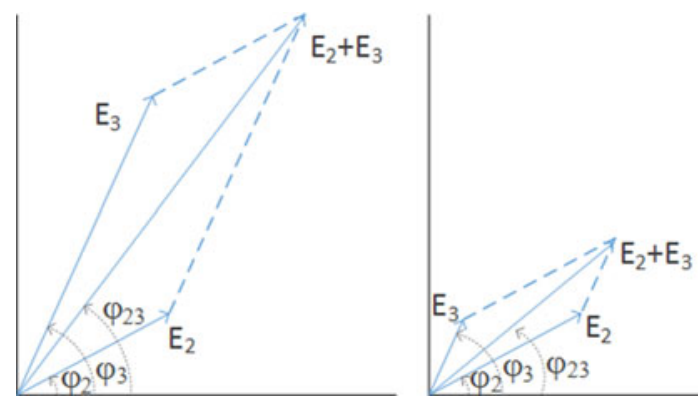

Fig. 2. Scheme of the resultant wave phase with the amplitude of $E_{3}$. In both cases, $E_{2}$ is constant and $E_{3}$ varies only in amplitude.

When the sensing head is placed in water, due to the smaller refractive index difference between the silica and the surrounding, $E_{3}$ presents substantially reduced amplitude. In such situation, $E_{2}$ cannot be neglected. Thus, both $E_{2}$ and $E_{3}$ interfere generating a new wave with a phase that depends on the relative phases of the two primary waves, as well as on their relative amplitudes. This interference, $E_{2}+E_{3}$, can be described through the equation:

$$
E_{2}+E_{3}=E_{023} \sin \left(\omega t+\varphi_{23}\right)
$$

where the amplitude $E_{023}$ is obtained from

$$
E_{023}=\left[E_{02}+E_{03}+2 E_{02} E_{03} \cos \left(\varphi_{2}-\varphi_{3}\right)\right]^{1 / 2} .
$$

In this case, $E_{02}$ and $E_{03}$ correspond to the amplitudes of the waves $E_{2}$ and $E_{3}$, respectively. The phase $\varphi_{23}$ is calculated according to the following equation:

$$
\varphi_{23}=\arctan \left[\frac{E_{02} \sin \varphi_{2}+E_{03} \sin \varphi_{3}}{E_{02} \cos \varphi_{2}+E_{03} \cos \varphi_{3}}\right] .
$$

In order to better understand the dependence of the resultant wave phase with the amplitude of $E_{3}$, a schematic diagram is shown in Fig. 2. In the diagram, only the amplitude of $E_{3}$ varies. Its phase is kept constant as well as the amplitude and phase of $E_{2}$. There is a clear modification on the interferometric wave when the amplitude of $E_{3}$ is reduced, both in amplitude and phase. Taking the equations presented previously in consideration, the spectral simulation of the resulting interferometric wave was obtained (see Fig. 3). Besides the clear variation in amplitude, the inset of Fig. 3 also shows the phase shift that occurs when the external medium is liquid.

This means the phase of this wave depends on the temperature induced variation of the refractive index of water because such variation originates a change of the silica-water reflectivity coefficient. The interference of this wave with $E_{1}$ translates into a global interference pattern with a phase that changes with the variation of the water refractive index, implementing in the optical domain the principle of the amplitude-phase conversion, i.e., the phase of the net interferometric optical signal becomes function of the amplitude of one of the interfering waves $\left(E_{3}\right)$. 


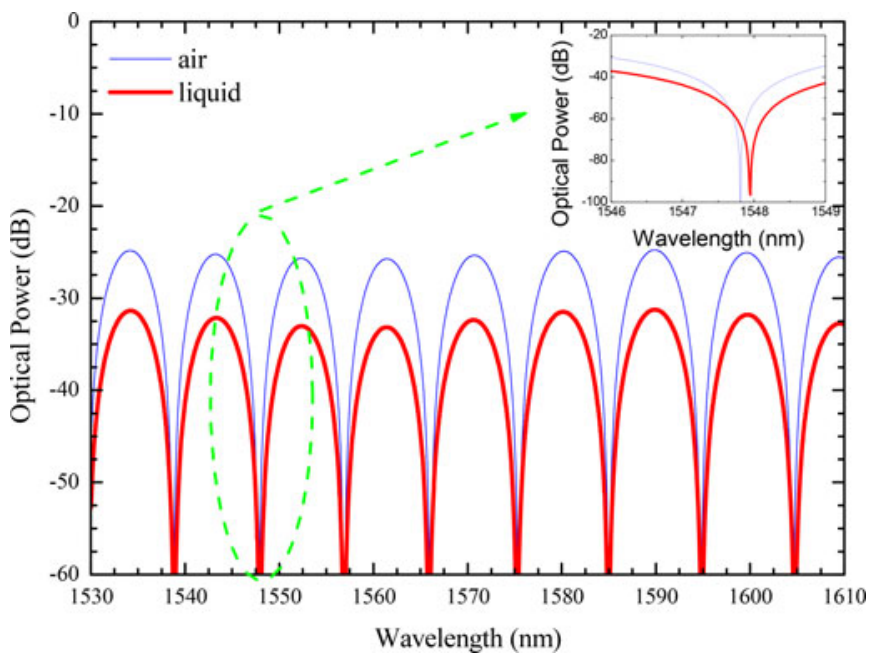

Fig. 3. Spectra of the FP microcavity in different media. The inset shows the phase variation of the spectrum.

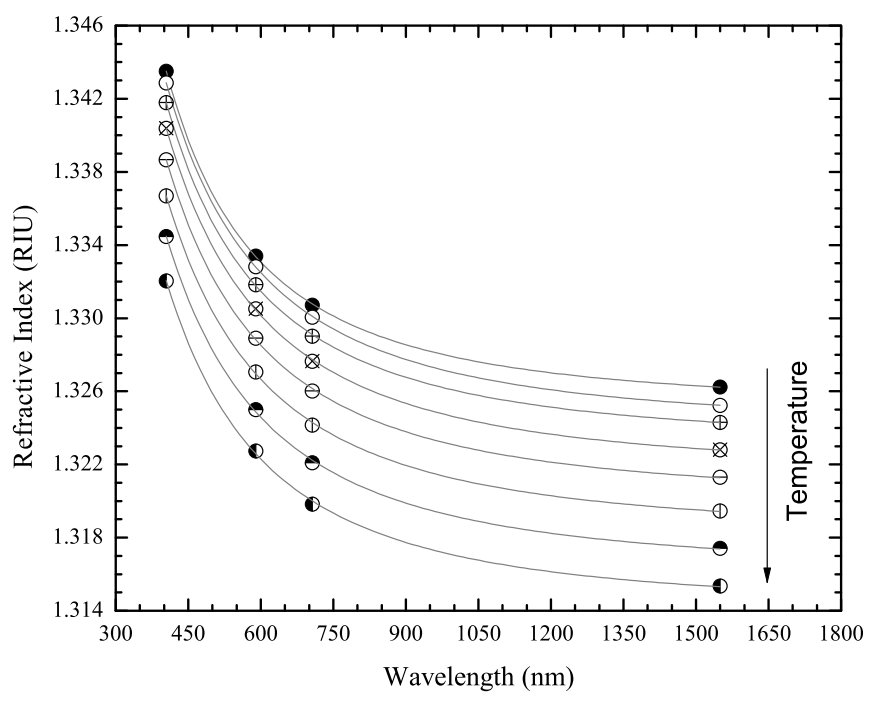

Fig. 4. Water refractive index variation with wavelength for different temperatures.

\section{B. Water Temperature and Refractive Index Relationship}

Several works have described the variation of the water refractive index with temperature [22]-[24]. However, the water refractive index depends not only on the temperature but also on the operation wavelength [25]. In the literature, tables were found for three different wavelengths: 430, 600, and $660 \mathrm{~nm}$. So, the first step is to estimate the refractive index at each considered temperature at $1550 \mathrm{~nm}$. The behavior can be described by the Sellmeier equation:

$$
n(\lambda)=A+\frac{B}{\lambda^{2}}+\frac{C}{\lambda^{4}}+\cdots
$$

where only the first three terms were taken into account. Using values reported in [25], a curve tendency can be adjusted as seen in Fig. 4. Notice that each curve is obtained at one given temperature. The considered temperatures ranged from $10^{\circ} \mathrm{C}$

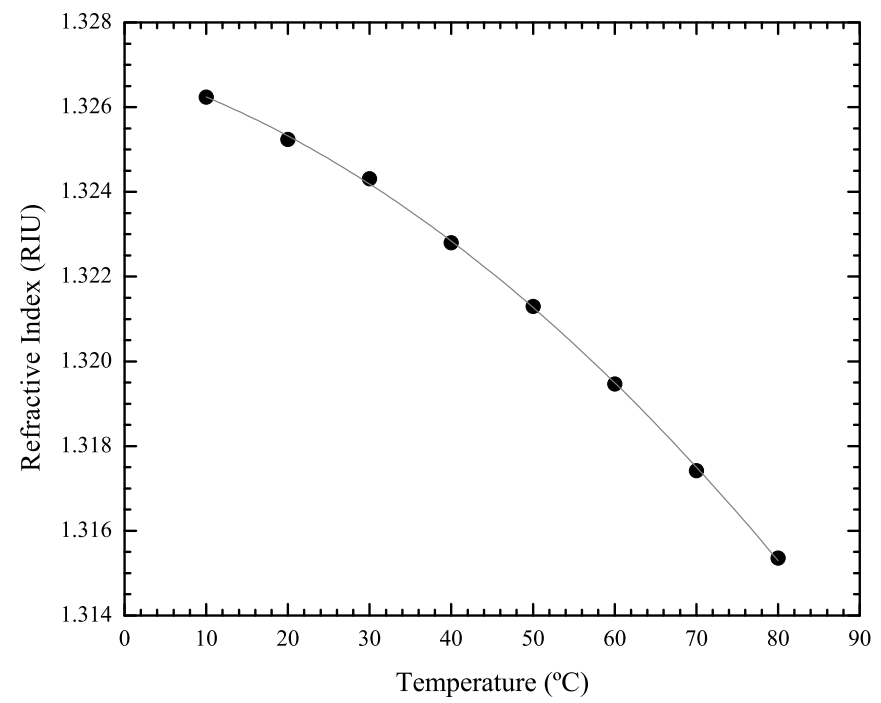

Fig. 5. Water refractive index variation with temperature at a wavelength of $1550 \mathrm{~nm}$.

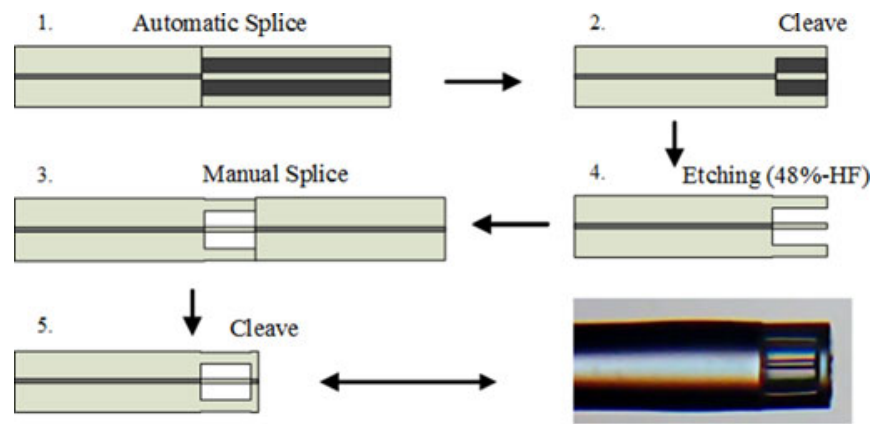

Fig. 6. Scheme of the optical fiber tip design fabrication steps.

to $80^{\circ} \mathrm{C}$ with a step of $10^{\circ} \mathrm{C}$. Through the adjusted equation, the refractive index at an operating wavelength of $1550 \mathrm{~nm}$ was obtained for different temperatures. The calculated values can be plotted as a function of temperature (see Fig. 5). The data are well adjusted by the second order polynomial:

$$
n(T)=-1.287 \times 10^{-6} T^{2}-3.802 \times 10^{-5} T+1.326 .
$$

Thus, by converting the water temperature in its refractive index variation, it is possible to observe the sensing head response towards this parameter. This is valid if the sensing head exhibits a response where the different contributions for its sensitivity can be discriminated.

\section{OPTICAL FIBER TIP DESIGN}

The phosphorous-doped DCF used in this study has been described in [16]. Initially, due to the higher refractive index in the inner cladding, no light will travel inside the fiber core. However, after applying the post-processing, light will propagate throughout the core. The optical fiber tips were produced by splicing a few hundred micrometers long section of P-doped DCF to SMF 28 (see steps 1 and 2 in Fig. 6). Afterwards, the tip was immersed in a solution of hydrofluoric acid (HF) with 


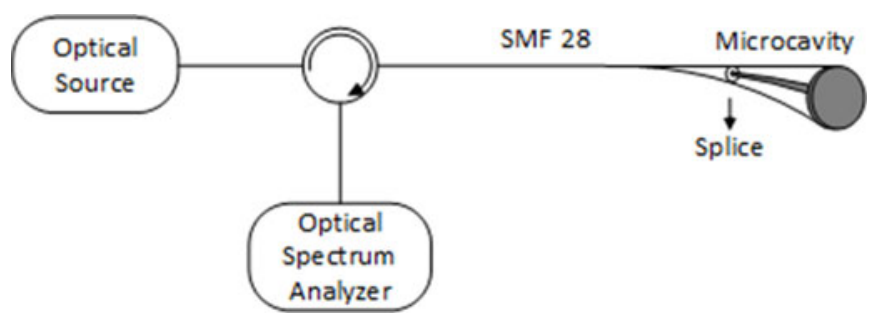

Fig. 7. Scheme of the experimental setup.

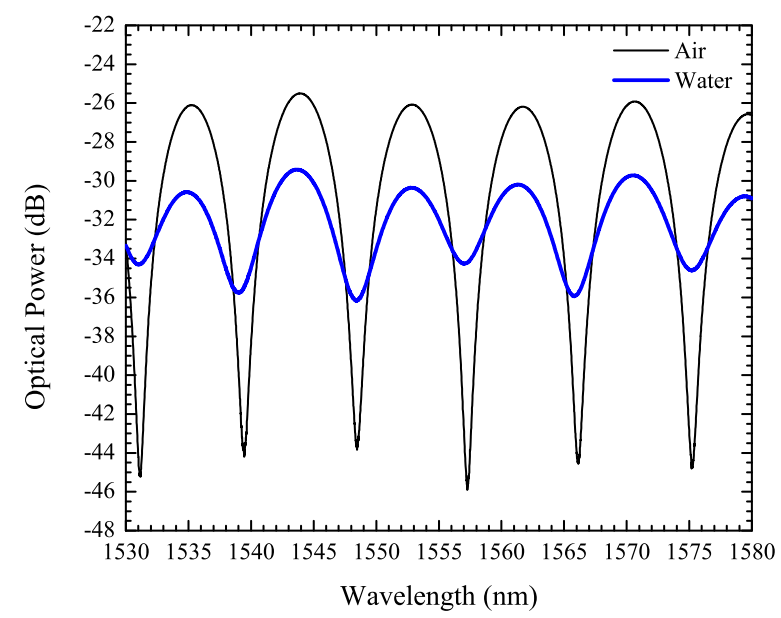

Fig. 8. Experimental spectra of the optical microcavity when in air (black solid line) and in water (bold blue line).

$48 \%$ concentration (step 3). In order to increase the etching rate, the HF solution was placed inside an ultrasound bath. Due to the presence of phosphorus inside the inner cladding, this region will be etched faster than the non-doped layers, creating a structure with a suspended core surrounded by air and protected by the outer cladding. This structure was then spliced to SMF 28 using a manual program of the splice machine [26] (step 4). Finally, the SMF 28 was cut closely to the DCF to form a diaphragm with a thickness of $\sim 15 \mu \mathrm{m}$ (step 5). The cleaving was performed under a $5 \times$ magnifying glass, enabling the control of the diaphragm thickness with accuracy lower than $10 \mu \mathrm{m}$.

The presence of the diaphragm is of most importance for the stability of the sensing head response when submerged in a liquid, as it guarantees that the liquid does not surround the DCF suspended core, which would cause instability in the spectral response.

\section{EXPERIMENTAL RESULTS}

The experimental setup, exhibited in Fig. 7, was constituted by a broadband optical source centered at $1570 \mathrm{~nm}$, with a bandwidth of $100 \mathrm{~nm}$, an optical circulator and an optical spectrum analyzer used to measure the reflection signal. The measurements were done with a resolution of $0.02 \mathrm{~nm}$. The DCF cavity used to perform the measurements had a length of $\sim 80 \mu \mathrm{m}$ and a core diameter of $\sim 8 \mu \mathrm{m}$. The diaphragm was $\sim 15 \mu \mathrm{m}$ long. Fig. 8 presents the sensing head spectra in air and in water.
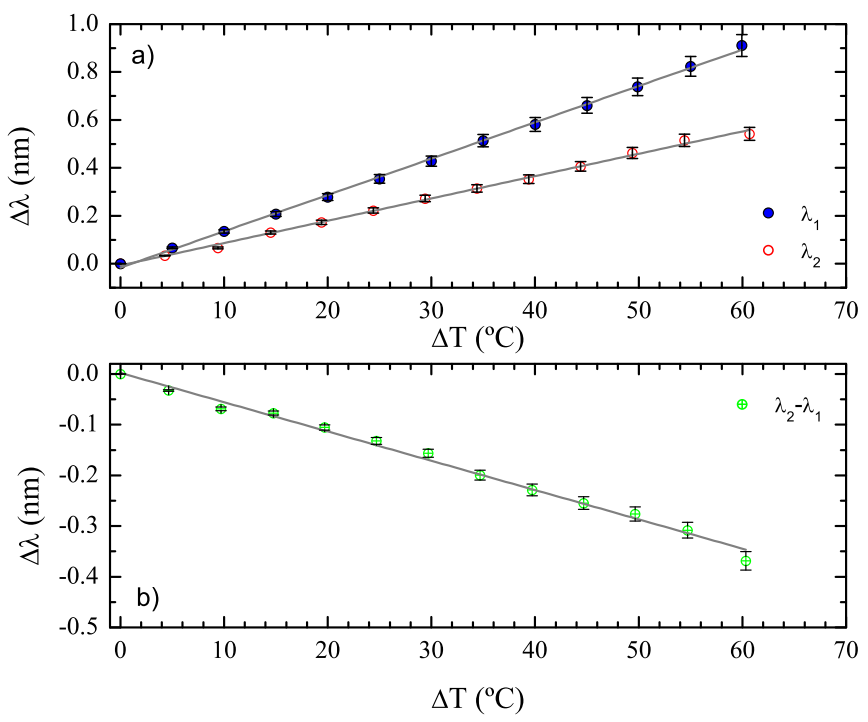

Fig. 9. Wavelength shift dependence with temperature: (a) sensing head exposed to air (solid circles) and when immersed in water (open circles) and (b) calculated water contribution.

These experimental data are in agreement with the simulation results presented in Fig. 3.

To test the sensing head, first it was placed in air inside a tubular oven. Measurements were done in a range between room temperature $\left(23^{\circ} \mathrm{C}\right)$ and $85^{\circ} \mathrm{C}$. There is a wavelength shift toward higher wavelengths (red shift) caused by the diaphragm silica thermal expansion [see Fig. 9(a)]. The sensitivity in this case is of $13.5 \mathrm{pm} /{ }^{\circ} \mathrm{C}$. The sensing head was also immerged in water in the same temperature range and a wavelength shift was observed as the temperature changed. The response, also presented in Fig. 9(a), continues to be well adjusted by a linear fitting and a sensitivity of $9.4 \mathrm{pm} /{ }^{\circ} \mathrm{C}$ was calculated. The difference between these values is an indication of the effect of the temperature on the refractive index of water has impact on the phase of the sensing optical cavity. The error bars exhibited in the Fig. 9 evidence the reproducibility of the results.

Therefore, if $\Delta \lambda_{1}$ is the wavelength shift of the cavity optical spectrum when the external medium is air, then we can write $\Delta \lambda_{1}=k_{T 1} \Delta T$, where $k_{\mathrm{T} 1}$ stands for the temperature sensitivity. If, on the other hand, the external medium is water the wavelength shift can be separated in two components: one due to the diaphragm silica thermal expansion $\left(k_{\mathrm{T} 1}\right)$, which was measured previously, and the other attributed to the water refractive index variation $\left(k_{\mathrm{T} 2}\right)$. Thus, the wavelength shift in this situation can be obtained through the expression $\Delta \lambda_{2}=\left(k_{T 1}+k_{T 2}\right) \Delta T$. Considering the two previous equations, the sensitivity due to the water contribution can be determined through $\Delta \lambda_{2}-\Delta \lambda_{1}=k_{T 2} \Delta T$.

Fig. 9(b) presents the calculated wavelength shift due the water contribution. The sensitivity is, in this case, negative, with a value of $-5.78 \mathrm{pm} /{ }^{\circ} \mathrm{C}$. By applying the relationship between the water refractive index and the temperature described in the theoretical section, it is possible to estimate the sensing head response to the variation of the external medium refractive index, as can be observed in Fig. 10. 


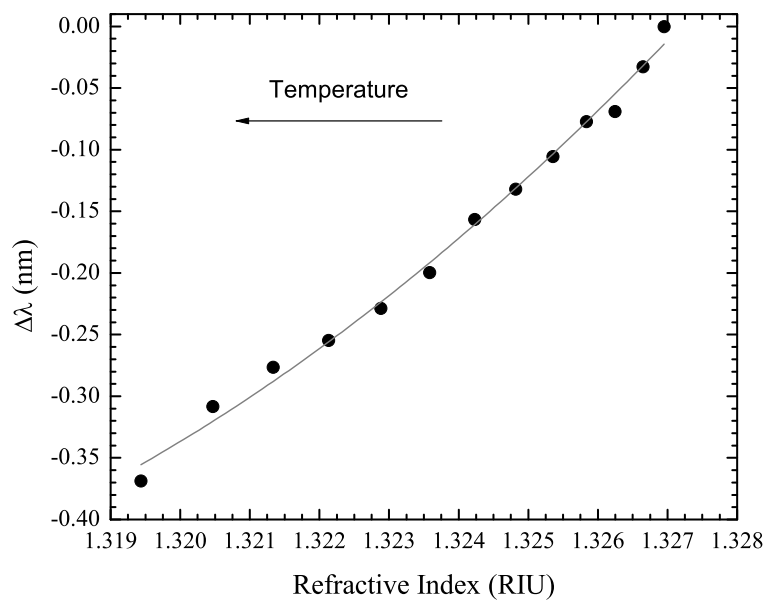

Fig. 10. Wavelength shift variation with the water refractive index.

By diminishing the temperature, the refractive index increases, and with it, there is a wavelength shift of the interferometric microcavity toward red. This shift was also adjusted to a second order polynomial,

$$
\lambda(n)=1758.70 n^{2}-4608.80 n+3018.92 .
$$

Two different linear regions can be extrapolated from this figure, for lower refractive indices (between 1.319 RIU and 1.324 RIU) and higher refractive indices (1.325 RIU to $1.327 \mathrm{RIU})$. A sensitivity of $38.70 \mathrm{~nm} / \mathrm{RIU}$ was obtained for the former, while a sensitivity of $54.68 \mathrm{~nm} / \mathrm{RIU}$ was obtained for the last region. Even though the sensitivities obtained are lower than the ones reported in the literature, it should be highlighted that there is no core exposition of the fiber to the external medium. The interaction between the third reflection wave at the end of the fiber tip ensures the spectrum phase variation.

It should also be noted that the results are highly dependent on the diaphragm thickness. For diaphragms with higher lengths ( $>30 \mu \mathrm{m}$ ), no change in the spectrum was observed when the sensing head was placed in water.

Besides the wavelength shift with the variation of the water temperature, a change in visibility was also noticed, due to the already mentioned change in refractive index. This indicates that a different kind of analysis could also be done with this sensing head. Besides, the sensing head was also subjected to high temperatures (up to $1000^{\circ} \mathrm{C}$ ) in air. The response was similar to the one obtained in [16], however, the correlation coefficient in this study was of 0.9973 whereas the one obtained in the mentioned reference was of 0.9983 . Even though the diaphragm-free sensor presented better adjustment, the proposed sensor can also be used in extreme environments.

\section{CONCLUSION}

In summary, a fiber microcavity three-wave interferometric sensor based on the post-processing of a DCF was proposed. Due to the presence of a diaphragm constituted by a short section of SMF, the sensing head was not only stable when submerged in water, but also sensible to is temperature variation.
When compared to the temperature measurements performed in air, the sensitivity in water became lower. This behavior is due to the fact that the sensing head was measuring, besides the silica thermal expansion, the water refractive index variation with temperature. In addition to these applications, the sensor can be used in a multiplexed configuration, allowing to compensate temperature or even to measure different parameters.

\section{REFERENCES}

[1] M. Deng, C.-P. Tang, T. Zhu, Y.-J. Rao, L.-C. Xu, and M. Han, "Refractive index measurement using photonic crystal fiber-based Fabry-Perot interferometer," Appl. Opt., vol. 49, no. 9, pp. 1593-1598, Mar. 2010.

[2] M. S. Ferreira, L. Coelho, K. Schuster, J. Kobelke, J. L. Santos, and O. Frazão, "Fabry-Perot cavity based on a diaphragm-free hollow-core silica tube," Opt. Lett., vol. 36, no. 20, pp. 4029-4031, Oct. 2011.

[3] D. Duan, Y. Rao, and T. Zhu, "High sensitivity gas refractometer based on all-fiber open-cavity Fabry-Perot interferometer formed by large lateral offset splicing," J. Opt. Soc. Amer. B., vol. 29, no. 5, pp. 912-915, May 2012.

[4] O. Frazão, J. M. Baptista, K. L. Santos, J. Kobelke, and K. Schuster, "Refractive index tip sensor based on Fabry-Perot cavities formed by a suspended core fibre," J. Eur. Opt. Soc., vol. 4, pp. 09041-1-09041-4, Aug. 2009.

[5] Y.-J. Rao, M. Deng, D.-W. Duan, and T. Zhu, "In-line fiber Fabry-Perot refractive-index tip sensor based on endlessly photonic crystal fiber," Sens. Actuators A, vol. 148, pp. 33-38, Jul. 2008.

[6] H. Y. Choi, G. Mudhana, K. S. Park, U.-C. Paek, and B. H. Lee, "Cross-talk free and ultra-compact fiber optic sensor for simultaneous measurement of temperature and refractive index," Opt. Exp., vol. 18, pp. 141-149, Jan. 2010.

[7] D. J. J. Hu, Y. Wang, J. L. Lim, T. Zhang, K. B. Milenko, Z. Chen, M. Jiang, G. Wang, F. Luan, P. P. Shum, Q. Sun, H. Wei, W. Tong, and T. R. Wolinski, "Novel miniaturized Fabry-Perot refractometer based on a simplified hollow-core fiber with a hollow silica sphere tip," IEEE Sensors J., vol. 12, no. 5, pp. 1239-1245, May 2012.

[8] O. Frazão, P. Caldas, J. L. Santos, P. V. S. Marques, C. Turck, D. J. Lougnot, and O. Soppera, "Fabry-Perot refractometer based on an endof-fiber polymer tip," Opt. Lett., vol. 34, no. 16, pp. 2474-2476, Aug. 2009.

[9] L. Rindorf and O. Bang, "Highly sensitive refractometer with a photoniccrystal-fiber long-period grating," Opt. Lett., vol. 33, pp. 563-565, Mar. 2008.

[10] K. Zhou, Z. Yan, L, Zhang, and I. Bennion, "Refractometer based on fiber Bragg grating Fabry-Perot cavity embedded in a narrow microchannel," Opt. Exp., vol. 19, no. 12, pp. 11769-11779, Jun. 2011.

[11] Z. L. Ran, Y. J. Rao, W. J. Liu, X. Liao, and K. S. Chiang, "Lasermicromachined Fabry-Perot optical fiber tip sensor for high-resolution temperature-independent measurement of refractive index," Opt. Exp., vol. 16, no. 3, pp. 2252-2263, Feb. 2008.

[12] C. R. Liao, T. Y. Hu, and D. N. Wang, "Optical fiber Fabry-Perot interferometer cavity fabricated by femtosecond laser micromachining and fusion splicing for refractive index sensing," Opt. Exp., vol. 20, pp. 22813-22817, Sep. 2012.

[13] L. V. Nguyen, M. Vasiliev, and K. Alameh, "Three-wave fiber Fabry-Perot interferometer for simultaneous measurement of temperature and water salinity of seawater," IEEE Phot. Tech. Lett., vol. 23, no. 7, pp. 450-452, Apr. 2011.

[14] K. Zhou, Y. Lai, X. Chen, K. Sugden, L. Zhang, and I. Bennion, "A refractometer based on a micro-slot in a fiber Bragg grating formed by chemically assisted femtosecond laser processing," Opt. Exp., vol. 15, pp. 15848-15853, Nov. 2007.

[15] H. Y. Choi, K. S. Park, S. J. Park, U.-C. Paek, B. H. Lee, and E. S. Choi, "Miniature fiber-optic high temperature sensor based on a hybrid structured Fabry-Perot interferometer," Opt. Lett., vol. 33, no. 21, pp. 24552457, Nov. 2008.

[16] M. S. Ferreira, J. Bierlich, S. Unger, K. Schuster, J. L. Santos, and O. Frazão, "Post-processing of Fabry-Perot microcavity tip sensor," IEEE Photon. Technol. Lett., vol. 25, no. 16, pp. 1593-1596, Aug. 2013.

[17] T. Wei, Y. Han, H.-L. Tsai, and H. Xiao, "Miniaturized fiber inline FabryPerot interferometer fabricated with a femtosecond laser," Opt. Lett., vol. 33, no. 6, pp. 536-538, Mar. 2008. 
[18] S. Silva, J. L. Santos, F. X. Malcata, J. Kobelke, K. Schuster, and O. Frazão, "Optical refractometer based on large-core air-clad photonic crystal fibers," Opt. Lett., vol. 36, pp. 852-854, Mar. 2011.

[19] Y. Wang, D. N. Wang, C. R. Liao, T. Hu, J. Guo, and H. Wei, "Temperatureinsensitive refractive index sensing by use of micro Fabry-Perot cavity based on simplified hollow-core photonic crystal fiber," Opt. Lett., vol. 38, pp. 269-271, Feb. 2013.

[20] Y. Chen and H. F. Taylor, "Multiplexed fiber Fabry-Perot temperature sensor system using white-light interferometry," Opt. Lett., vol. 27, no. 11, pp. 903-905, Jun. 2002.

[21] L. Liu, Y. Gong, Y. Wu, T. Zhao, H.-J. Wu, and Y.-J. Rao, "Spatial frequency multiplexing of fiber-optic interferometric refractive index sensors based on graded-index multimode fibers," Sensors, vol. 12, pp. 1237712385, Sep. 2012.

[22] G. S. Kell, "Precise representation of volume properties of water at one atmosphere," J. Chem. Eng. Data, vol. 12, no. 1, pp. 66-69, Jan. 1967.

[23] G. Abbate, U. Bernini, E. Ragozzino, and F. Somma, "The temperature dependence of the refractive index of water," J. Phys. D, Appl. Phys., vol. 11, pp. 1167-1172, Jan. 1978

[24] J. D. Olson and F. H. Horne, "Direct determination of temperature dependence of refractive index of liquids," J. Chem. Phys., vol. 58, no. 6, pp. 2321-2325, Mar. 1973.

[25] I. Thormählen, J. Straub, and U. Grigull, "Refractive index of water and its dependence on wavelength, temperature and density," J. Phys. Chem. Ref. Data, vol. 14, no. 4, pp. 933-945, 1985.

[26] O. Frazão, J. P. Carvalho, and H. M. Salgado, "Low loss splice in a microstructured fibre using a conventional fusion splicer," Microw. Opt. Technol. Lett., vol. 46, pp. 172-174, Jul. 2005.

Marta S. Ferreira received the Graduate degree in physical engineering and the M.Sc. degree in 2009 from the University of Aveiro, Aveiro, Portugal. She is currently working toward the Ph.D. degree with the Optoelectronics and Electronic Systems Unit, INESC Porto, Portugal and the Faculty of Sciences, University of Porto, Porto. She has published several papers in international journals and conferences. Her research interests include post-processing of optical fibers, fiber sensing, and fiber lasers. She is a Student Member of the SPIE and OSA.

Jörg Bierlich received the Graduate degree in medical engineering from the University of Applied Sciences Jena, Germany, in 2001 and the Ph.D. degree focused on the preparation and characterization of superconducting ceramic composites from the University of Technology "Bergakademie" Freiberg, Germany, in 2008. He finished his professional training in 1994 as an Energy Electronics Engineer at JENOPTIK GmbH, Jena. From 2001 to 2008, he worked at the Department of Magnetics and Quantum Electronics, Institute of Photonic Technology (IPHT Jena and investigated high-temperature superconductors. Since 2008, he has been working at the Department of Fiber Optics, IPHT Jena in the field of fiber drawing technologies and the development of special optical fibers. He is involved in the preparation and characterization of microstructured fibers based on high silica and other glass materials.
Sonja Unger was born in Rathen, Germany, on May 4, 1951. She received the Ph.D. degree in inorganic chemistry from the College of Education, Erfurt, Germany. She is currently with the Institute of Photonic Technology (IPHT) Jena, Germany, as a Research Collaborator, and she is involved in research in the field of development of specialty optical fibers.

Kay Schuster is involved for many years in preparation of specialty fibers, based on heavy metal oxide glasses, chalcogenide glasses, and high purity silica. His research interests include the preparative operations of glass manufacturing as well as material characterization (DTA, TG, UV-VIS, FTIR spectroscopy, refractive index) and fiber fabrication (preform and crucible drawing). Based on his knowledge and experience both, special glass preparation and optical fiber drawing could be highly qualified at the Institute of Photonic Technology, Jena, Germany for different applications. Results of these activities were active non silica single mode fibers for amplification ( $\operatorname{Pr} 3+$ doped chalcogenide fibers for $1.3 \mu \mathrm{m}, \mathrm{Er}^{3+}$ doped $\mathrm{HMO}$ fibers for $1.5 \mu \mathrm{m}$ broadband amplification). His current research interests include design and preparation of special functionalized microstructured fibers for passive, active, and remote sensing applications. Very complex structures of this high silica and silicate glass based fibers have been realized. In the applicative critical field of special sensor fibers for high temperature applications, he is intensively involved in the application and alignmen of suitable fiber coating materials (e.g., ORMORCERs, siloxanes, polyimides, metals). Since 2012, he has been the Group Leader of the Optical Fibers Technology Group within the Fiber Optics Division. Beside the fabrication of specialty microstructured fibers, the group is intensively involved in preform and fiber manufacturing for high power fiber lasers and FBG and Raman based sensing applications.

José L. Santos received the Graduate degree in physics (optics and electronics) from the University of Porto, Porto, Portugal, in 1983, and the Ph.D. and Habilitation degrees in 1993 and 2008, respectively, from the same University. His main research interests include optical fiber sensing and optical fiber technology. He is a Full Professor with Physics and Astronomy Department, University of Porto and is a Researcher with Optoelectronics and Electronic Systems Unit, INESC Porto. He is a Member of the OSA, SPIE, and Planetary Society.

Orlando Frazão received the Graduate degree in physical engineering from the University of Aveiro, Aveiro, Portugal, and the Ph.D. degree in physics from the University of Porto, Porto, Portugal. From 1997 to 1998, he was with the Institute of Telecommunications, Aveiro. He is currently an invited Assistant Professor with the Department Physics, Faculty of Science, University of Porto and a Senior Researcher with Optoelectronics and Electronic Systems Unit, INESC Porto. His current research interests include optical fiber sensors and optical communications. He has more than 300 papers in international journals and conferences. He participated as organized committee of several International conferences. He has three scientific awards. He is the Senior Member of the SPIE and OSA. 